\title{
Electronic and magnetic properties of zinc blende half-metal superlattices
}

C. Y. Fong, M. C. Qian, J. E. Pask, L. H. Yang, and S. Dag

Citation: Appl. Phys. Lett. 84, 239 (2004); doi: 10.1063/1.1639934

View online: http://dx.doi.org/10.1063/1.1639934

View Table of Contents: http://aip.scitation.org/toc/apl/84/2

Published by the American Institute of Physics

\section{Articles you may be interested in}

Possible half-metallic ferromagnetism in zinc blende $\mathrm{CrSb}$ and $\mathrm{CrAs}$ (invited)

Journal of Applied Physics 93, 6844 (2003); 10.1063/1.1558604

Room-temperature ferromagnetism in zincblende $\mathrm{CrSb}$ grown by molecular-beam epitaxy

Applied Physics Letters 79, 2776 (2001); 10.1063/1.1413732

Investigation of spin-gapless semiconductivity and half-metallicity in Ti2MnAl-based compounds

Applied Physics Letters 108, 141901 (2016); 10.1063/1.4945600

Antiferromagnetic half-metals, gapless half-metals, and spin gapless semiconductors: The $\mathrm{D}_{3}$-type Heusler alloys

Applied Physics Letters 103, 232409 (2013); 10.1063/1.4840318

Search for spin gapless semiconductors: The case of inverse Heusler compounds

Applied Physics Letters 102, 022402 (2013); 10.1063/1.4775599

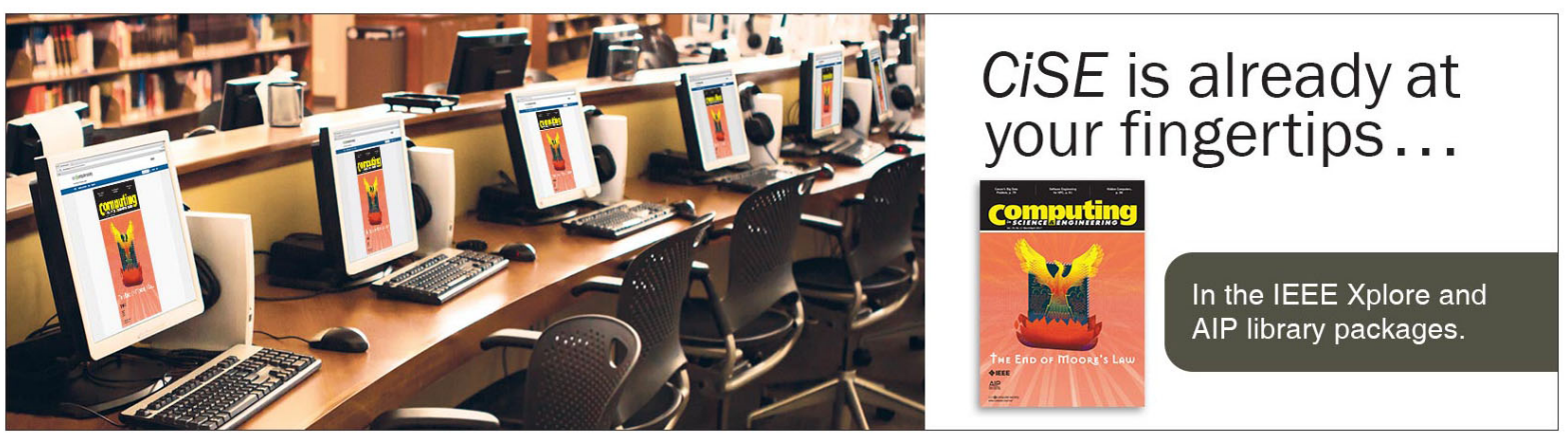




\title{
Electronic and magnetic properties of zinc blende half-metal superlattices
}

\author{
C. Y. Fong and M. C. Qian \\ Department of Physics, University of California, Davis, California 95616 \\ J. E. Pask and L. H. Yang ${ }^{\text {a) }}$ \\ Lawrence Livermore National Laboratory, Livermore, California 94551
}

S. Dag

Department of Physics, Bilkent University, Ankara 06533, Turkey

(Received 23 September 2003; accepted 19 November 2003)

\begin{abstract}
Zinc blende half-metallic compounds such as CrAs, with large magnetic moments and high Curie temperatures, are promising materials for spintronic applications. We explore layered materials, consisting of alternating layers of zinc blende half-metals, by first principles calculations, and find that superlattices of $(\mathrm{CrAs})_{1}(\mathrm{MnAs})_{1}$ and $(\mathrm{CrAs})_{2}(\mathrm{MnAs})_{2}$ are half-metallic with magnetic moments of $7.0 \mu_{B}$ and $14.0 \mu_{B}$ per unit cell, respectively. We discuss the nature of the bonding and half-metallicity in these materials and, based on the understanding acquired, develop a simple expression for the magnetic moment in such materials. We explore the range of lattice constants over which half-metallicity is manifested, and suggest corresponding substrates for growth in thin film form. (C) 2004 American Institute of Physics. [DOI: 10.1063/1.1639934]
\end{abstract}

With the successful syntheses of half-metallic (HM) zinc blende (ZB) CrAs in thin film form by Akinaga et al., ${ }^{1}$ a unique class of spintronic materials has been born. This success has stimulated further work to discover and understand other such transition metal compounds ${ }^{2-5}$ and layered structures. ${ }^{6-8}$ Of particular interest for spintronic applications, $\mathrm{HM} \mathrm{Cr}$ and $\mathrm{Mn}$ pnictides have been found to possess large magnetic moments: $3.0 \mu_{B}$ for $\mathrm{Cr}$ and $4.0 \mu_{B}$ for $\mathrm{Mn}$ compounds, per formula unit.

Here, we investigate layered materials composed of alternating layers of $\mathrm{HM}$ ZB CrAs and MnAs: $(\mathrm{CrAs})_{1}(\mathrm{MnAs})_{1}$ and $(\mathrm{CrAs})_{2}(\mathrm{MnAs})_{2}$. In particular, we investigate whether half-metallicity is preserved in the layered structures, whether the magnetic moment is preserved, the nature of the bonding and half-metallicity, and possible substrates for growth.

We employed an ultrasoft pseudopotential ${ }^{9}$ planewave density functional ${ }^{10}$ approach, ${ }^{11}$ with generalized gradient approximation ${ }^{12}$ to exchange and correlation. This approach has been shown to yield excellent agreement with allelectron calculations for the compounds of interest here. ${ }^{5}$ Metal $3 d$ and $4 s$, and As $4 s$ and $4 p$ states were included in valence. A planewave cutoff of $450 \mathrm{eV}$ was used in all calculations. Increasing the planewave cutoff from 450 to 650 $\mathrm{eV}$ resulted in changes in total energy of less than $10^{-3} \mathrm{eV}$ in CrAs and MnAs calculations. The one-layer and two-layer structure Brillouin zones were sampled using 1859 and $726 k$ points, ${ }^{13}$ respectively. Increasing the number of $k$ points from 726 to 2925 resulted in changes in total energy of less than $2 \times 10^{-3} \mathrm{eV}$ in two-layer structure calculations. Lattice constants were optimized, and atomic positions were relaxed to within $0.06 \mathrm{eV} / \AA$ in all cases.

Two layered structures, $(\mathrm{CrAs})_{1}(\mathrm{MnAs})_{1}$ and $(\mathrm{CrAs})_{2}(\mathrm{MnAs})_{2}$, were considered. The conventional cell of

a)Electronic mail: 1yang@1lnl.gov the $(\mathrm{CrAs})_{1}(\mathrm{MnAs})_{1}$ structure is shown in Fig. 1. The primitive cell is tetragonal with $1 \mathrm{Cr}, 1 \mathrm{Mn}$, and $2 \mathrm{As}$ atoms. The ideal $\mathrm{ZB}$ position of the $\mathrm{Cr}$ atom is at the lower left corner, represented by a small shaded circle. Mn and As atoms are indicated by filled and open circles, respectively. The optimized lattice constant $(5.70 \AA)$ is close to the average of the optimized lattice constants of the constituent CrAs (5.66 $⿱$ ) and MnAs $(5.77 \AA)$ compounds. ${ }^{5}$ This lattice constant applies also to the two-layer structure.

The calculated total and projected densities of states (DOS) for the $(\mathrm{CrAs})_{1}(\mathrm{MnAs})_{1}$ structure are shown in Fig. 2. The structure is half-metallic at its optimized lattice constant, though only one of its constituents (CrAs) is ( $\mathrm{ZB}$ MnAs being only nearly half-metallic at its equilibrium lattice constant). The DOS shows much in common with those of the constituent compounds. ${ }^{5}$ Considering first the majority states, we find low-lying, isolated As- $s$ states at $\sim-10 \mathrm{eV}$, followed by As- $p$-metal- $d$ hybridized states in the vicinity of $E_{F}$. Mn states dominate the $d$ manifold at lower energies, while $\mathrm{Cr}$ states dominate at higher, consistent with nuclear charges. The metallicity is contributed mainly by $\mathrm{Cr} d$ states. The minority $d$ states are shifted significantly relative to the majority by the exchange interaction, with the more localized, nonbonding $e_{g}$ states shifted substantially more than

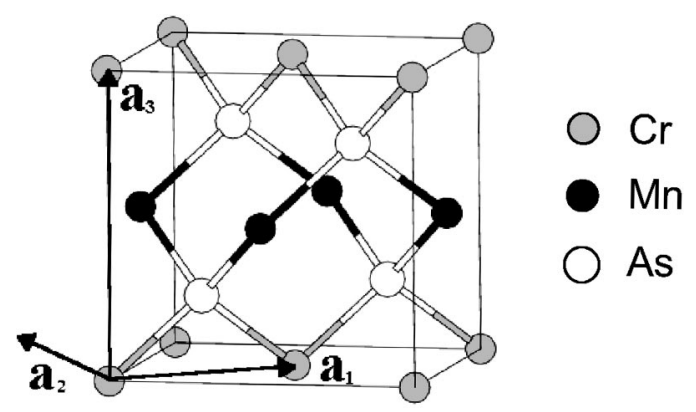

FIG. 1. (CrAs $)_{1}(\mathrm{MnAs})_{1}$ superlattice conventional cell. 


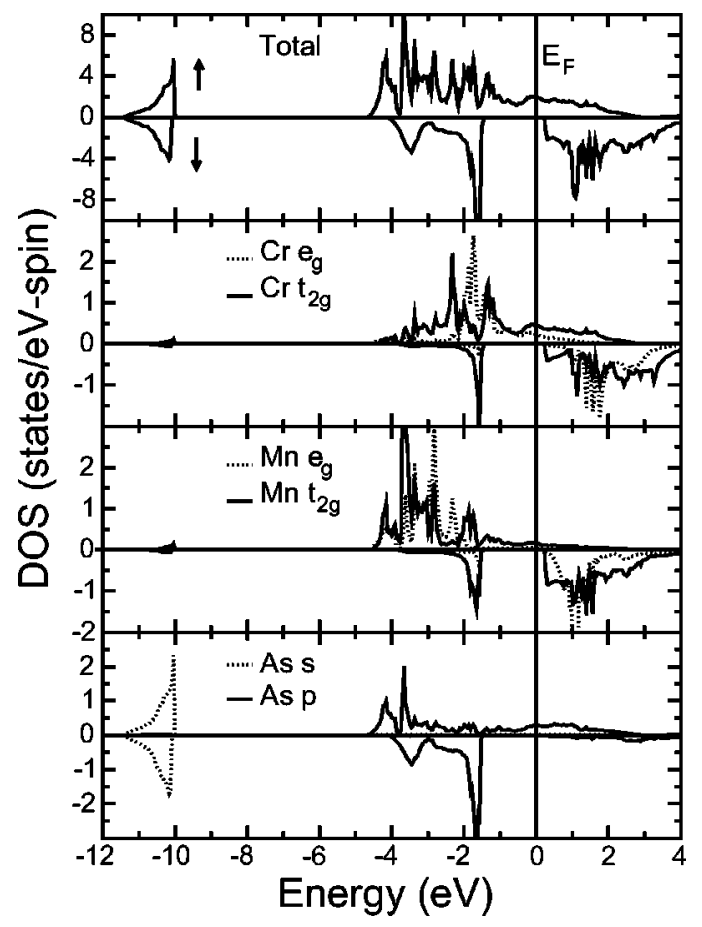

FIG. 2. Calculated total and projected densities of states of $(\mathrm{CrAs})_{1}(\mathrm{MnAs})_{1}$ at the optimized lattice constant. The material is halfmetallic. As- $p$ and metal- $d$ states show significant hybridization, and $\mathrm{Cr} d$ states contribute most strongly to the metallicity.

the strongly hybridized, bonding $t_{2 g}$ states, opening a gap at $E_{F}$. Thus, as in the constituent compounds, the bonding is mainly As- $p$-metal- $d$ in nature and the minority-spin gap is opened up by virtue of the substantially differing exchange splitting of $t_{2 g}$ and $e_{g}$ manifolds. No interface states form in the gap, and half-metallicity is manifested. The same general features are exhibited in the two-layer structure. A comparison of key features of the electronic structure of both layered structures and constituent compounds is given in Table I.

The calculated majority and minority valence charge densities in a plane containing a $\mathrm{Cr}-\mathrm{As}-\mathrm{Mn}-\mathrm{As}$ chain are shown in Fig. 3. The majority states show As- $p-\mathrm{Mn}-d$ bonding, consistent with the hybridization exhibited in the majority DOS (Fig. 2). Also clear is the substantial, strongly localized $e_{g}$ density at the metal atoms (with lobes along the vertical axis), consistent with substantially lesser hybridization. The minority states also show As- $p-\mathrm{Mn}-d$ bonding. However, here the specific $t_{2 g}$ character at the metal atom is apparent, consistent with the shift of the $e_{g}$ states completely

TABLE I. Calculated half-metallic properties of $(\mathrm{CrAs})_{1}(\mathrm{MnAs})_{1}$ and $(\mathrm{CrAs})_{2}(\mathrm{MnAs})_{2}$ superlattices, and constituent ZB compounds. DOS at $E_{F}$ is the density of states at the Fermi energy in the majority channel, $E_{g}$ is the gap in the minority channel, and $M$ is the magnetic moment. All results are per primitive unit cell.

\begin{tabular}{lccc}
\hline \hline \multicolumn{1}{c}{ Sample } & $\begin{array}{c}\text { DOS at } E_{F} \\
\text { (states/eV-spin) }\end{array}$ & $E_{g}(\mathrm{eV})$ & $M\left(\mu_{B}\right)$ \\
\hline$(\mathrm{CrAs})_{1}(\mathrm{MnAs})_{1}$ & 1.94 & 1.65 & 7.0 \\
$(\mathrm{CrAs})_{2}(\mathrm{MnAs})_{2}$ & 3.47 & 1.62 & 14.0 \\
$\mathrm{CrAs}^{\mathrm{a}}$ & 0.85 & 1.85 & 3.0 \\
$\mathrm{MnAs}^{\mathrm{a}}$ & 0.77 & 1.70 & 4.0 \\
\hline \hline
\end{tabular}

${ }^{\mathrm{a}}$ Ref. 5.

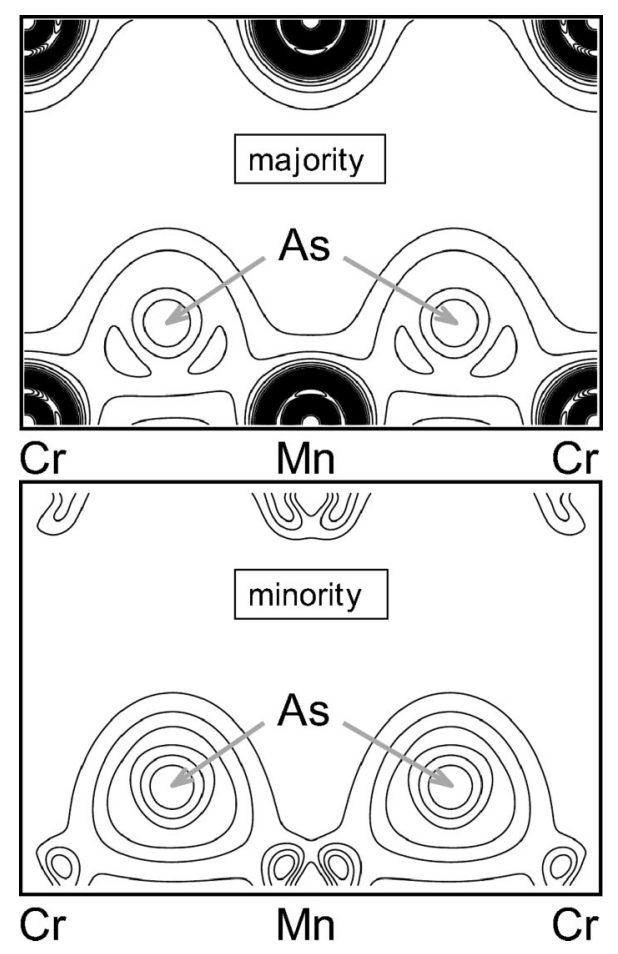

FIG. 3. Calculated majority- and minority-spin valence charge densities of $(\mathrm{CrAs})_{1}(\mathrm{MnAs})_{1}$ in a plane containing a $\mathrm{Cr}-\mathrm{As}-\mathrm{Mn}-\mathrm{As}$ chain. Contours are equally spaced in both plots. Both majority and minority densities show As- $p$-metal- $d$ bonding. The majority density shows substantial, strongly localized $e_{g}$ character at the metal atoms, notably absent in the minority density which exhibits clear $t_{2 g}$ character.

above $E_{F}$ by the exchange interaction, as manifested in the associated $t_{2 g}$ DOS (Fig. 2).

Because the layered materials are half-metallic and share a common minority electronic structure with the constituent compounds (As- $s$ and As- $p$-metal- $t_{2 g}$ hybrid states completely filled, metal- $e_{g}$ and all higher states completely unfilled), a simple relation may be developed for their magnetic moments, analogous to that for the constituent compounds. Proceeding as in Ref. 5, we have then

$$
M=\left(Z_{\mathrm{tot}}-2 N_{\text {min }}\right) \mu_{B}=\left(Z_{\mathrm{tot}}-8 N_{\mathrm{As}}\right) \mu_{B}
$$

for the magnetic moment per unit cell, $M$; where $Z_{\text {tot }}$ is the total number of valence electrons, $N_{\min }$ is the number of occupied minority states, and $N_{\mathrm{As}}$ is the number of As atoms, per unit cell. The $8 N_{\mathrm{As}}$ on the right-hand side of Eq. (1) follows from the fact that each minority As- $s$ state holds one electron while each minority As- $p$-metal $t_{2 g}$ hybrid state holds three, for a total of four minority electrons per As atom. For the $(\mathrm{CrAs})_{1}(\mathrm{MnAs})_{1}$ layered structure, $Z_{\mathrm{tot}}=23$ and $N_{\mathrm{As}}=2$, and so Eq. (1) predicts a magnetic moment of $7 \mu_{B}$ per unit cell; and similarly, a magnetic moment of $14 \mu_{B}$ per unit cell for $(\mathrm{CrAs})_{2}(\mathrm{MnAs})_{2}$. The ab initio calculations confirm exactly these values (Table I).

Of course, the increased magnetic moments for larger cells implied by Eq. (1) do not necessarily imply increased saturation magnetizations (magnetic moment per unit volume). For ZB CrAs, with a magnetic moment of $3.0 \mu_{B}$ per unit cell (Table I), the saturation magnetization is $572.4 \mathrm{emu} / \mathrm{cm}^{3}$, which compares well with the measured value of $559.8 \mathrm{emu} / \mathrm{cm}^{3}{ }^{1}$ For $\mathrm{ZB}$ MnAs, it is $763.2 \mathrm{emu} / \mathrm{cm}^{3}$. The saturation magnetization for both super- 
lattices is $672.8 \mathrm{emu} / \mathrm{cm}^{3}$, approximately the average of the two constituents, due to the preserved constituent-like minority electronic structure in the layered materials. More generally, we find that the magnetic moment per unit cell of the layered materials is the sum of the magnetic moments per unit cell of the constituents, due to the preserved minority electronic structure. And so it is not viable to modify the saturation magnetization of such materials by adding layers; there is at most a small volume effect.

To determine possible substrates for growth of these layered materials, we carried out calculations for a range of lattice constants. We find that between 5.60 and $6.03 \AA$, the superlattices retain their integer magnetic moments (a necessary condition of half-metallicity). This range spans the experimental lattice constants of AlAs (5.62 $⿱$ ) $),$ GaAs (5.65 $\AA)$, InAs $(6.04 \AA)$, and $\operatorname{InP}(5.81 \AA) .{ }^{14}$ Growth with minimal strain might therefore be accomplished on AlAs, GaAs, or InP.

In summary, we find layered structures of $(\mathrm{CrAs})_{1}(\mathrm{MnAs})_{1}$ and $(\mathrm{CrAs})_{2}(\mathrm{MnAs})_{2}$ to be half-metallic using ab initio electronic structure methods. We find the bonding and half-metallicity are fundamentally the same as in the constituent ZB compounds; and further find that due to the nature of the bonding, the magnetic moment per unit cell of such materials can be predicted exactly by a simple expression [Eq. (1)]. These materials are expected to grow in thin film form, as have ZB CrAs and MnSb. We determined the range of lattice constants consistent with half-metallicity in these materials and suggested corresponding substrates for growth.
C.Y.F. acknowledges support from NSF Grants Nos. ESC-0225007 and INT-9872053, and NERSC at Lawrence Berkeley National Laboratory. This work was performed, in part, under the auspices of the U.S. DOE by the University of California, Lawrence Livermore National Laboratory under Contract No. W-7405-Eng-48. The authors gratefully acknowledge the support of the Materials Research Institute at LLNL.

${ }^{1}$ H. Akinaga, T. Manago, and M. Shirai, Jpn. J. Appl. Phys., Part 2 39, L1118 (2000).

${ }^{2}$ A. Continenza, S. Picozzi, W. T. Geng, and A. J. Freeman, Phys. Rev. B 64, 085204 (2001).

${ }^{3}$ S. Sanvito and N. A. Hill, Phys. Rev. B 62, 15553 (2000).

${ }^{4}$ I. Galanakis and P. Mavropoulos, Phys. Rev. B 67, 104417 (2003).

${ }^{5}$ J. E. Pask, L. H. Yang, C. Y. Fong, W. E. Pickett, and S. Dag, Phys. Rev. B 67, 224420 (2003).

${ }^{6}$ M. Shirai, T. Ogawa, I. Kitagawa, and N. Suzuki, J. Magn. Magn. Mater. 177-181, 1383 (1998).

${ }^{7}$ S. Sanvito and N. A. Hill, Phys. Rev. Lett. 87, 267202 (2001).

${ }^{8}$ P. Mavropoulos, I. Galanakis, and P. Dederichs, arXiv:condmat/0304403 (2003).

${ }^{9}$ D. Vanderbilt, Phys. Rev. B 41, 7892 (1990).

${ }^{10}$ P. Hohenberg and W. Kohn, Phys. Rev. 136, B864 (1964); W. Kohn and L. J. Sham, Phys. Rev. 140, A1133 (1965).

${ }^{11}$ G. Kresse and J. Hafner, J. Phys.: Condens. Matter 6, 8245 (1994); G. Kresse and J. Furthmüller, Phys. Rev. B 54, 11169 (1996).

${ }^{12}$ See, e.g., K. Burke, J. P. Perdew, and Y. Wang, in Electronic Density Functional Theory: Recent Progress and New Directions, edited by J. F. Dobson, G. Vignale, and M. P. Das (Plenum, New York, 1998).

${ }^{13}$ H. J. Monkhorst and J. D. Pack, Phys. Rev. B 13, 5188 (1976).

${ }^{14}$ R. W. G. Wyckoff, Crystal Structures, 2nd ed. (Interscience, New York, 1963), Vol 1. 